\title{
Stroke Epidemiology: Advancing Our Understanding of Disease Mechanism and Therapy
}

\author{
Bruce Ovbiagele • Mai N. Nguyen-Huynh
}

Published online: 21 June 2011

(C) The American Society for Experimental NeuroTherapeutics, Inc. 2011

\begin{abstract}
Stroke is the fourth killer and number one cause of adult disability in the United States. The estimated direct and indirect costs of stroke care in this country are $\$ 68.9$ billion for 2009. The prevalence of stroke and its cost will undoubtedly rise as the aging population increases. In addition, stroke incidence and mortality are increasing in less developed countries in which the lifestyles and population restructuring are rapidly changing. More population-based research to assess incidence, risk factors, and outcomes are needed in these countries. Epidemiologic studies can help identify groups of individuals or regions at higher risk for stroke. They can also help us better understand the natural history of certain conditions and therefore push the direction of therapeutic investigations. Furthermore, the study of trends across different time periods and different populations can help investigators evaluate the effects of stroke care programs and treatment options.
\end{abstract}

Keywords Stroke · Review. Epidemiology· Outcome · Mortality. Incidence

Electronic supplementary material The online version of this article (doi:10.1007/s13311-011-0053-1) contains supplementary material, which is available to authorized users.

B. Ovbiagele

Department of Neurology, University of California, San Diego, La Jolla, CA 92093, USA

M. N. Nguyen-Huynh $(\bowtie)$

Stroke Sciences Group, University of California,

San Francisco, CA 94143, USA

e-mail: mai.n.nguyen-huynh@ucsfmedctr.org

\section{Introduction}

Epidemiologic studies on stroke help us with understanding the natural history of the disease, identification of risk factors, and prognostic factors that can lead to markers for disease mechanisms. Epidemiology and observational data can inform scientists of possible novel areas for more focused research. They help us identify the individuals, groups, or geographic areas that are at increased risk of disease or poorer outcomes. Critical analyses of the factors that are involved in those cases of higher risks and poorer outcomes can lead to a better understanding of the underlying causes. Furthermore, knowing which individuals, groups, or geographic areas are at risk helps physicians and public health policymakers to develop more effective target programs to reduce the risk of stroke, stroke mortality, and disability.

\section{Prevalence and Incidence}

Stroke, a highly prevalent condition, exacts a substantial societal toll in the form of the "Dreaded-D's," which are the leading cause of chronic disability, the second leading cause of dementia, and the fourth leading cause of death in the United States (U.S.) [1]. The worldwide impact of stroke seems to be even more pronounced than it is in the U.S., but overall estimates vary widely and may not necessarily be consistently reliable [2]. In the U.S., the prevalence of stroke is roughly $3 \%$ of the adult population, which translates to approximately 7 million individuals [1].

Approximately 800,000 primary (first-time) or secondary (recurrent) strokes occur each year in the U.S., with the majority being primary strokes (roughly 600,000) [1]. Of these strokes, approximately $87 \%$ are ischemic infarctions, $10 \%$ are primary hemorrhages, and $3 \%$ are subarachnoid 
hemorrhage. Worldwide estimates indicate that primary hemorrhages constitute a higher percentage of all strokes, ranging from $10 \%$ to $25 \%$. Individuals of Asian, African, and Latin American origin tend to have a higher frequency of primary hemorrhage than persons of European origin [1]. In addition, although primary hemorrhage accounts for 10 to $17 \%$ of all strokes in Western countries, in Asian it is approximately $25 \%$ [3].

The incidence of stroke rapidly increases with age, doubling for each decade after age 55 [4]. Among adults ages 35 to 44 , the incidence of stroke is 30 to 120 of 100,000 per year, and for those ages 65 to 74 , the incidence is 670 to 970 of 100,000 per year [1]. Stroke does occur among children, but the incidence in comparison with adults is substantially lower (i.e., approximately 1 to 2.5 of 100,000 per year), and roughly 50 to $75 \%$ of strokes among children are as a result of hemorrhage [1]. Sickle cell disease is the most common cause of childhood stroke, with the highest incidence between ages 2 to 5 years [5].

Perhaps due to better control of vascular risk factors during the last decade, incidence rates of stroke in developed nations have modestly diminished or plateaued. For instance, in the U.S. between 1997 and 2006, the total hospital-based stroke diagnoses decreased from 680,607 to 609,359 , and the age-adjusted hospital-based stroke diagnosis rate per 100,000 persons significantly declined in a linear pattern from 282.7 to 210.4 in men $(26 \%)$ and from 240.5 to 184.7 in women (23\%) [6]. The average rate of decrease in hospital-based stroke diagnosis rates was significantly greater in men than in women $(-8.7$ vs -7.5 per 100,000 persons). It has been projected that with continued aging of the population, especially in Western countries, these rates are expected to rise again dramatically during the next 40 years.

A transient ischemic attack (TIA) is classically viewed as a temporary abrupt-onset neurological deficit due to brain or retinal ischemia lasting less than 24 hours in duration. Determining the exact incidence or prevalence of TIAs can be challenging because symptoms experienced can sometimes be neurologically nonspecific, due to its transitory nature. Many patients do not seek medical attention, and for those who do seek help, it is in a delayed fashion with symptom characteristics that become hazy with time. As such, data on TIA prevalence broadly varies, but the range of 1 to $6 \%$ with prevalence in the U.S. is estimated to be $2.3 \%$ [7]. Several population-based studies have investigated the issue of TIA incidence. A study (in Rochester, Minnesota) observed a crude age- and sex-adjusted incidence rate of 68 per 100,000 persons per year for the years 1985 to 1989 , the highest incidence of 584 per 100,000 noted among individuals aged 75 to 84 [8]. Other population-based TIA incidence rates range from 18 per 100,000 persons per year from 1987 to 1988 in Novosibirsk,
Russia [9] to 37 per 100,000 persons per year from 19701973 in Estonia [10]. Data from Sweden, England, France, and Japan have also been within this range [11-14]. The higher rates in the Rochester study may reflect distinct methods of case ascertainment because incidence rates for ischemic stroke are comparable. Generally, incidence rates of classically defined TIA incidence rates have remained stable with time.

An expert consensus group recently recommended a formal change in the definition of TIA to a "transient episode of neurological dysfunction caused by focal brain, spinal cord, or retinal ischemia, without acute infarction," thereby eliminating the time-based component noted in the classical definition [15]. The decision to consider adopting a tissue-based (not an arbitrary timebased) definition of TIA was primarily driven by modern imaging data, which revealed that as many as $50 \%$ of patients with transient deficits lasting $<24$ hours have evidence of brain ischemia on initial magnetic resonance imaging, and $50 \%$ of those with initial abnormalities on magnetic resonance imaging show evidence of fixed infarction on subsequent images. Estimates suggest that the potential epidemiologic impact of adopting a tissuebased definition of TIA in the U.S. would lower annual incidence rates of TIA by $33 \%$ from roughly 180,000 to 120,000 , and would increase the rate of ischemic stroke by this amount [16].

Another change in our approach to TIA stems from a better understanding of its short-term prognosis. It is now clear that that the risk of future vascular events within 3 months is as high or higher after TIA, as after completed stroke, and this risk is highest within the first week after TIA [17]. Comparisons across studies point to a 90-day stroke risk ranging from 10 to $20 \%$ after TIA [17]. In response to this new knowledge, prediction scores have been developed and validated to facilitate better decisionmaking in the evaluation of imminent risk of stroke after TIA [18]. These scores could potentially be used to identify TIA patients who may require urgent admission, but have not yet been tested in a clinical trial.

Prevalence of asymptomatic cerebral ischemia noted on brain imaging among persons aged 55 to 64 years is $11 \%$, and this rises to $22 \%$ for those aged 65 to 69 years, $28 \%$ for those aged 70 to 74 years, $32 \%$ for those aged 75 to 79 years, $40 \%$ for those aged 80 to 85 years, and $43 \%$ for individuals $\geq 85$ years of age [1]. U.S. data revealed that $17.8 \%$ of the U.S. population $\geq 45$ years of age with no prior diagnosis of stroke or TIA reported at least 1 symptom of stroke. Prevalence of these strokerelated symptoms were more likely among blacks vs whites, persons with lower income and lower educational attainment, and those with fair to poor perceived health status [1]. 


\section{Risk Factors}

Prevention remains the most viable avenue for lessening the burden of stroke on society, particularly given the high incidence of stroke worldwide, insidious contribution of stroke risk factors, and the paucity of proven acute stroke therapies. Longitudinal studies have identified several characteristics/conditions that boost a person's risk for primary or recurrent stroke [4, 19]. These risk factors are viewed as nonmodifiable or modifiable and generally apply to primary and recurrent stroke. Nonmodifiable risk factors serve as markers for high stroke risk (Table 1) [4], whereas modifiable risk factors are amenable to intervention for lower stroke risk. A population-based study that compared two epochs (1981 to 1984 vs 2002 to 2004 in Oxfordshire, United Kingdom) observed a lowered population level in the prevalence of multiple risk factors (including smoking, elevated cholesterol, and high blood pressure) was linked with a reduction for stroke incidence. Recently, an international case-control study (i.e., the INTERSTROKE study) of 3000 stroke cases, and corresponding number of controls, showed that roughly $90 \%$ of strokes could be explained by 10 risk factors: 1) hypertension, 2) diabetes, 3) cardiac causes, 4) current smoking, 5) abdominal obesity, 6) hyperlipidemia, 7) physical inactivity, 8) alcohol consumption, 9) diet, and 10) psychosocial stress and depression [20]. Nonetheless, unexplained stroke risk and increasing realization that atherosclerosis is the culprit in most strokes is, in part, an inflammatory condition that has led to a search for novel modifiable stroke risk factors. Some of the potentially modifiable ischemic stroke risk factors being studied are listed in Table 2 [21, 22]. Risk factors for primary intracerebral hemorrhage are shown in Table 3 and overlap to some degree with those for ischemic stroke [3].

\section{Modifiable Medical Risk Factors}

Hypertension Hypertension is the most prominent modifiable risk factor for ischemic stroke, and it afflicts more than 75 million adults $\geq 20$ years of age in the U.S. [1]. Due to its widespread prevalence, depending on age group, the population attributable risk of hypertension for stroke is as great as $40 \%$ [4], and in the INTERSTROKE study, depending on the definition used, hypertension accounted for as great as $50 \%$ of the risk of stroke [20]. Hypertension prevalence varies by race ethnicity (highest in blacks), resulting in a differential effect of hypertension on stroke risk by race ethnicity. Elevated stroke risk has been linked with all stages of hypertension and isolated systolic hypertension [23]. In fact, the risk of stroke seems to have a continuous association with blood pressure down to levels as low as $115 / 75 \mathrm{mmHg}$ [23]. In light of this, the national guidelines redefined categories of hypertension so that normal systolic blood pressure is $<120 \mathrm{mmHg}$ and normal diastolic blood pressure is $<80 \mathrm{mmHg}$ [24]. Most recently, it has been suggested that the variability in blood pressure measurements (visit to visit, distinct measurements within a given visit) are associated with greater risk of stroke [25].

Atrial Fibrillation Chronic atrial fibrillation (AF) is a strong stroke risk factor and it is estimated to affect more than 2.6 million persons in the U.S. in 2010 [1]. Among individuals $>65$ years of age, the prevalence of AF is approximately $6 \%$. Because the prevalence of AF rises with age, the attributable risk of stroke due to $\mathrm{AF}$ is highest in much older age groups [4]. So, for instance, AF may account for as much as $25 \%$ of strokes among persons aged 80 to 89 years. The risk of stroke is approximately 20 times higher among AF patients with valvular disease and five times higher among AF patients with nonvalvular disease

Table 1 Nonmodifiable risk factors for stroke

\begin{tabular}{ll}
\hline Risk factor & Impact on stroke incidence \\
\hline Age & Doubles for every successive decade after the age of 55 years \\
Sex & $24 \%$ to $30 \%$ higher in men; however, absolute annual number of women experiencing stroke is higher because women \\
& outlive men \\
Race ethnicity & 2- to 4-fold higher among African Americans and occur at an earlier age \\
& 2-fold higher among Hispanics and occur at an earlier age \\
& Higher among Chinese \\
& Higher rates in the Southeastern U.S. (the so-called "Stroke Belt"), especially along the coasts in Georgia and the Carolinas \\
Geography & (so-called "Stroke Buckle"). \\
& Almost 2-fold higher among first-degree relatives. Chromosome 9p21 (proximal to genes CDKN2A and CDKN2B) has been \\
Heredity & linked to ischemic stroke risk
\end{tabular}

${ }^{a}$ Compared to non-Hispanic whites 
Table 2 Promising modifiable risk factors for ischemic stroke being studied

\begin{tabular}{|c|c|}
\hline Risk entity & Possible risk factors \\
\hline \multicolumn{2}{|c|}{ Inflammation } \\
\hline & - High sensitivity C-reactive protein \\
\hline & - Lipoprotein-associated phospholipase A2 (LpPLA2) \\
\hline & - Peripheral leukocyte count \\
\hline & - Other cytokines (interleukin 6 , tumor necrosis factor and its receptors) \\
\hline \multicolumn{2}{|l|}{ Infections } \\
\hline & - Chlamydia pneumonia \\
\hline & - Helicobacter pylori \\
\hline & - Herpes viruses \\
\hline & - Periodontal infections \\
\hline \multicolumn{2}{|c|}{ Renal Disease } \\
\hline & - Decreased glomerular filtration function (glomerular filtration rate, Cystatin C) \\
\hline & - Abnormal glomerular filtration barrier (albuminuria) \\
\hline \multicolumn{2}{|c|}{ Dietary Habit } \\
\hline & - High saturated fat intake \\
\hline & - Elevated salt intake \\
\hline
\end{tabular}

compared to patients without AF [26]. Clinical trial and epidemiological data have been used to derive various stroke risk stratification schemes that can be used in clinical practice for AF patients [26]. Of note, outpatient continuous arrhythmia monitoring is increasingly showing that AF may actually be responsible for a higher percentage of unexplained strokes than was previously known [27].

Coronary Artery Disease Individuals with a presence of coronary artery disease have double the risk of stroke compared to patients without coronary artery disease [19]. The attributable risk of stroke due to coronary artery disease is approximately $12 \%$. Coronary artery disease patients with left ventricular hypertrophy have 3 times the risk of stroke, whereas coronary artery disease patients with congestive heart failure have 4 times the risk [19]. Within 5 years of a myocardial infarction, the rate of stroke is $8.1 \%$ [28], and older patients or patients with a cardiac ejection fraction less than $28 \%$ are at higher risk of stroke.
Diabetes A population-based study of more than 14,000 subjects observed that the presence of diabetes was independently related to a greater risk of ischemic stroke (adjusted risk ratio, 2.26) [29]. In the Northern Manhattan Stroke Study (NOMASS), the prevalence of diabetes was as high as $22 \%$ among elderly black subjects, and as high as $20 \%$ among elderly Hispanics, with corresponding attributable stroke risks of $13 \%$ and $20 \%$, respectively [4]. Insulin resistance without the presence of overt diabetes is associated with a greater risk of stroke. In the Atherosclerosis Risk in Communities study, elevated fasting insulin levels in nondiabetics was related to higher risk of stroke (relative risk, 1.19 per $50 \mathrm{pmol} / \mathrm{L}$ rise). Furthermore, among nondiabetic subjects in NOMASS, those with elevated measurements of insulin resistance were significantly more likely to have a first ischemic stroke, even after adjusting for other risk factors and the metabolic syndrome [30]. The metabolic syndrome, a constellation of glucose dysmetabolism, obesity, hypertension, and dyslipidemia has been

Table 3 Major risk factors for spontaneous intracerebral hemorrhage ${ }^{\mathrm{a}}$

\begin{tabular}{ll}
\hline Risk Factor & Effect Size (95\% Confidence Interval) [3] \\
\hline Hypertension & Odds Ratio: $3.68(2.52-5.38)$ \\
Age (every 10 year rise) & Risk Ratio: $1.97(1.79-2.16)$ \\
Current smoking & Odds Ratio: $1.31(1.09-1.58)$ \\
Diabetes Mellitus & Odds Ratio: $1.30(1.02-1.67)$ \\
High alcohol intake $(>56$ grams/day) & Odds Ratio: $4.11(2.54-6.65)$ \\
Moderate alcohol intake $(<56$ grams/day) & Odds Ratio: $2.05(1.35-3.11)$
\end{tabular}

${ }^{a}$ Other risk factors include drug abuse (e.g., cocaine, amphetamines), excessive anticoagulation, trauma, low serum cholesterol, thrombolytic therapy, and revascularization procedures 
shown to independently confer greater risk of first and recurrent stroke [31, 32]. It is unclear if metabolic syndrome confers a greater risk of first stroke than what one would expect for its components [33].

Dyslipidemia Abnormalities in several serum lipid indices have been linked to symptomatic vascular disease. These associations have been particularly robust in regard to coronary artery disease, but at times it is conflicting in regard to stroke [4]. However, many early studies that examined the relationship of lipids with stroke only examined total serum cholesterol levels and did not include stroke subtyping. Not accounting for the heterogeneity of stroke pathophysiology ( $v s$ coronary artery disease), likely contributed to the inconsistent findings [4]. Recent studies that have addressed the limitations of prior studies generally have shown an association of elevated serum triglycerides, total cholesterol, low-density lipoprotein cholesterol, and nonhigh-density lipoprotein cholesterol with ischemic stroke risk, especially atherosclerotic and lacunar stroke subtypes. For instance, in 1 study the highest total cholesterol quintile was related to greater ischemic stroke risk $v s$ the lowest quintile (odds ratio, 1.6; 95\% confidence interval, 1.3 to 2.0 ), and the most robust subtype associations were for atherosclerotic stroke (OR, 3.2) and lacunar stroke (OR, 2.4) [34]. Elevated high-density lipoprotein cholesterol was shown to be protective for stroke in NOMASS [4].

Asymptomatic Carotid Stenosis Prevalence of asymptomatic carotid stenosis rises with age, and can be found in more than $50 \%$ of individuals 65 years of age or older. Earlier studies found the risk of stroke with asymptomatic carotid stenosis to be approximately $1.3 \%$ per year among patients with stenosis less or equal to $75 \%$, and approximately $3.3 \%$ annually among patients with stenosis greater than 75\% [35]. The "best" medical therapy has changed because the publications of clinical trials have compared carotid endarterectomy with medical therapy for asymptomatic carotid stenosis [36]. The risk of stroke associated with asymptomatic carotid stenosis has fallen significantly during the past 20 years [36, 37]. With contemporary medical therapy, the average annual rate of ipsilateral stroke is estimated to be $<1 \%$.

\section{Modifiable Lifestyle Risk Factors}

Cigarette Smoking Smoking is associated with reduced blood vessel distensibility/compliance, elevated fibrinogen levels, increased platelet aggregation, decreased highdensity lipoprotein cholesterol levels, and higher hematocrit [4]. The relative risk of stroke for smokers included in a large meta-analysis was 1.5 , and a dose-response associa- tion with higher stroke risk has been observed in heavy $v s$ light smokers. Roughly $18 \%$ of strokes are attributable to active cigarette smoking. Stroke risk associated with former smoking has been shown to substantially decrease with increasing time because of cessation, and the Framingham study found stroke risk to be at the level of nonsmokers at 5 years from cessation. Even passive cigarette smoking boosts progression of atherosclerosis. Indeed, there is a greater risk of ischemic stroke (relative risk, 4.8) among cigarette-smoking women with a cigarette-smoking spouse vs those with a nonsmoking spouse. Finally, smoking modifies the influence of oral contraceptives on stroke risk, as there seems to be a 7-fold rise in risk among persons who both smoke and use oral contraceptives [38].

\section{Alcohol Consumption}

Heavy alcohol consumption is associated with elevated blood pressure, enhanced coagulability, cardiac arrhythmias, and decrease in cerebral blood flow [4]. On the other hand, light-to-moderate consumption has been linked to elevated high-density cholesterol and endogenous tissue plasminogen activator levels. Increasing alcohol consumption is associated with greater risk of hemorrhagic stroke in a dose-dependent manner. However, studies evaluating the impact of alcohol consumption on ischemic stroke risk have not shown consistent results. Indeed, the majority of published evidence points to a protective effect of light-tomoderate drinking (1-2 drinks per day) on the risk of ischemic stroke including data from the Nurses' Health Study and NOMASS (adjusted odds ratio, 0.5).

Physical Inactivity Increased physical activity is associated with reductions in fibrinogen, homocysteine, and platelet activity, as well as elevations in high-density lipoprotein cholesterol and plasma tissue plasminogen activator activity [4]. Observational data show that physical activity is linked with lower stroke risk, whereas sedentary behavior is related to higher stroke risk. A meta-analysis of 23 studies that examined the relationship of physical activity with risk of stroke noted that highly active subjects experienced a $27 \%$ lower risk of stroke or mortality vs low-active subjects.

\section{Stroke Outcomes}

Stroke is the fourth leading cause of death in the U.S. and the number 1 cause of long-term disability worldwide. It is estimated that someone dies of a stroke every 4 minutes in the U.S. [39]. In 2006, approximately $47 \%$ of stroke deaths occurred in the hospital [40]. The 1-month case fatality rate 
in the U.S. for all strokes ranges from $10 \%$ to $15 \%$ [41-44]. The case fatality rate is lower for ischemic stroke $(8-12 \%)$ compared to hemorrhagic stroke (33-45\%) [43-45]. There are approximately 6.4 million stroke survivors living in the U.S. The estimated direct and indirect cost of stroke for 2007 is $\$ 62.7$ billion [46]. At 6 months after stroke, 35\% had depressive symptoms, $30 \%$ were unable to ambulate without assistance, and $26 \%$ were dependent in activities of daily living [1]. Understanding the factors that are associated with clinical outcomes can lead to more effective interventions to reduce case fatality and mortality, and to improve functional status in patients with stroke.

\section{Effect of Age on Outcomes}

Stroke incidence increases with age [39]. With prolonged life expectancy, we can expect to see an increasing number of older patients presenting with stroke. How age influences stroke outcome has been the subject of a number of studies [43-45, 47, 48]. Many have found age to be associated with poorer outcomes, independent of stroke type. In general, older patients were more likely to be discharged to an institution other than home, and were more disabled and more severely handicapped at 3 months after stroke [47, 48]. Those $\geq 80$ years old with stroke had a longer length of stay compared to younger patients $(24.7 \pm 27.6$ vs $22.3 \pm$ 24.2 days; $p=0.013$ ) [48]. In-hospital mortality and case fatality also increase with age $[47,48]$. Case fatality rate for those $\geq 80$ years old can be as high as $21 \%$ [48]. Although age seems to influence activities of daily living recovery, it has not been found to have an effect on neurological recovery [47]. This may suggest that older patients have a poorer ability to compensate, and therefore may benefit more from rehabilitation with a focus on activities of daily living.

\section{Effect of Gender on Outcomes}

Gender differences in heart disease are well recognized, but less is known of gender differences in stroke. Although age-adjusted stroke mortality rates for men are higher than for women overall, due to their longevity, more women die of stroke each year than men, accounting for almost $61 \%$ of all stroke deaths in the U.S. in 2006 [39]. A number of studies have shown that women have more severe strokes than men [49]. It has also been estimated that women with acute ischemic stroke were $30 \%$ less likely to receive thrombolysis treatment with intravenous tissue plasminogen activator (tPA) than men [50]. Based on community studies, it has been found that women have a higher case of fatality rate than men, although confounders were not adjusted for all the studies. From 21 studies, the pooled case fatality for men was $19.7 \%$ and for women it was $24.7 \%$; therefore, it is 1.25 times higher among women $(95 \%$ confidence interval, 1.17 to 1.34 ) [49].

In addition, women who survive stroke seem to have less favorable outcomes than men. Women have longer length of stay and are less likely to be discharged home [51-54]. Female gender independently predicts poorer prognosis and less independence at 3 to 6 months poststroke, even after adjustment for age, comorbidities, and other clinical features $[51-53,55,56]$. The etiology of these differences is unclear. A large multi-center study across Europe has noted that gender, rather than stroke severity, was a major discriminating factor for the use of diagnostic neuroimaging studies or therapeutic interventions, such as carotid surgery [51]. More studies are needed to identify modifiable determinants of poorer stroke outcomes in women (such as usage of available diagnostic or therapeutic options, secondary stroke prevention measures, rehabilitation efforts, or social networks) so that targeted interventions can be instituted to reduce these disparities.

\section{Geographic Variations in Outcomes}

Many studies have described geographic variations in stroke mortality in the U.S., with higher rates seen in the Southeastern U.S. ("Stroke Belt") since at least the 1950s. Using data from the Medicare Enrollment Database, 1 study found that the overall recurrent ischemic stroke rates declined by almost 5\% from 1994 to 2002 [57]. However, this varied significantly by region, and there were counties in the Southeast U.S. who had major declines in stroke recurrence, but their rates generally remained greater than the national average. Globally, there are also regional differences in stroke mortality with a higher burden seen in North Asia, Eastern Europe, Central Africa, and the South Pacific [58]. The causes of such geographic variations remain unclear. One of the many hypothesized reasons is geographic variations in stroke risk factors. However, in the U.S., an analysis using risk factor data from the Reasons for Geographic and Racial Differences in Stroke (REGARDS) study showed that the risk factor hypothesis likely only explains a small percentage of the geographic variation for stroke mortality [59]. Globally, national income has been reported to be a strong predictor of stroke burden and mortality. However, this association did not change much with adjustments for typical vascular risk factors, which were more prevalent in high-income countries [58]. Other hypotheses to explain geographic variations include differences in socioeconomic status (SES), lifestyle choices, infection rates, other novel risk 
factors, access to care, and aggressiveness of care, but data to support these are lacking.

\section{Effect of Socioeconomic Status on Outcomes}

The relationship between poverty and health inequality has been demonstrated in many different countries and at different levels of national healthcare coverage [60-62]. What drives this relationship is unclear because comparisons between published studies are difficult to make due to the heterogeneity of the studies, including the choice of which indicator of SES to measure (income, education, occupation, class, and so forth). The fact remains that these proxy measures of SES have been shown to be inversely associated with stroke case fatality, poorer functional and motor recovery during rehabilitation, less health services after stroke, and increased recurrent stroke risk [62-65]. Stroke burden and mortality vary greatly geographically, but low-income countries are the most affected (Fig. 1) [58]. Some possible mechanisms by which SES variables are associated with disease outcomes are by creating differentials in the use of social support structures, healthcare systems, and access to knowledge and resources [62].

\section{Effect of Race Ethnicity on Outcomes}

In 2007 , the death rate for stroke was 40.2 per 100,000 for white males and 67.1 per 100,000 for black males, and 39.3 for white females and 55.0 for black females [1]. Mortality rates from intracerebral hemorrhage for blacks and Asian
Pacific Islanders (API) were 1.7 and 1.5 times higher than whites for 1995 to 1998 [66]. For subarachnoid hemorrhage during the same period, all ethnic groups had higher rates than whites.

Racial disparities in stroke incidence and outcomes have been well-described. Cultural and environmental mechanisms play a role in racial ethnic stroke disparities, including aspects of socioeconomic class, access to care, discrimination, and cultural variations in risk factors. There is strong evidence that blacks in the U.S. have higher stroke incidence and higher death rates for all stroke subtypes compared to other racial groups [39]. Asian Pacific Islanders have been found to have a higher death rate from intracerebral hemorrhage than whites [66]. The Brain Attack Surveillance in Corpus Christi project found Mexican Americans to have a higher risk of stroke recurrence than non-Hispanic whites, and found that this risk was strongly associated with a risk of death [67]. However, it is less clear what accounts for these types of differences.

The Brain Attack Surveillance in Corpus Christi project did not find a significant difference in ischemic stroke subtypes among Mexican Americans and non-Hispanic whites, and therefore they were unlikely to be strong predictors of differences observed in stroke incidence or mortality between these ethnic groups [68]. In recent years, it has become clear that intracranial atherosclerotic disease occurs at a much higher prevalence among Asians, blacks, and Hispanics [69]. The poor natural history of intracranial atherosclerotic disease likely contributes to the rates of

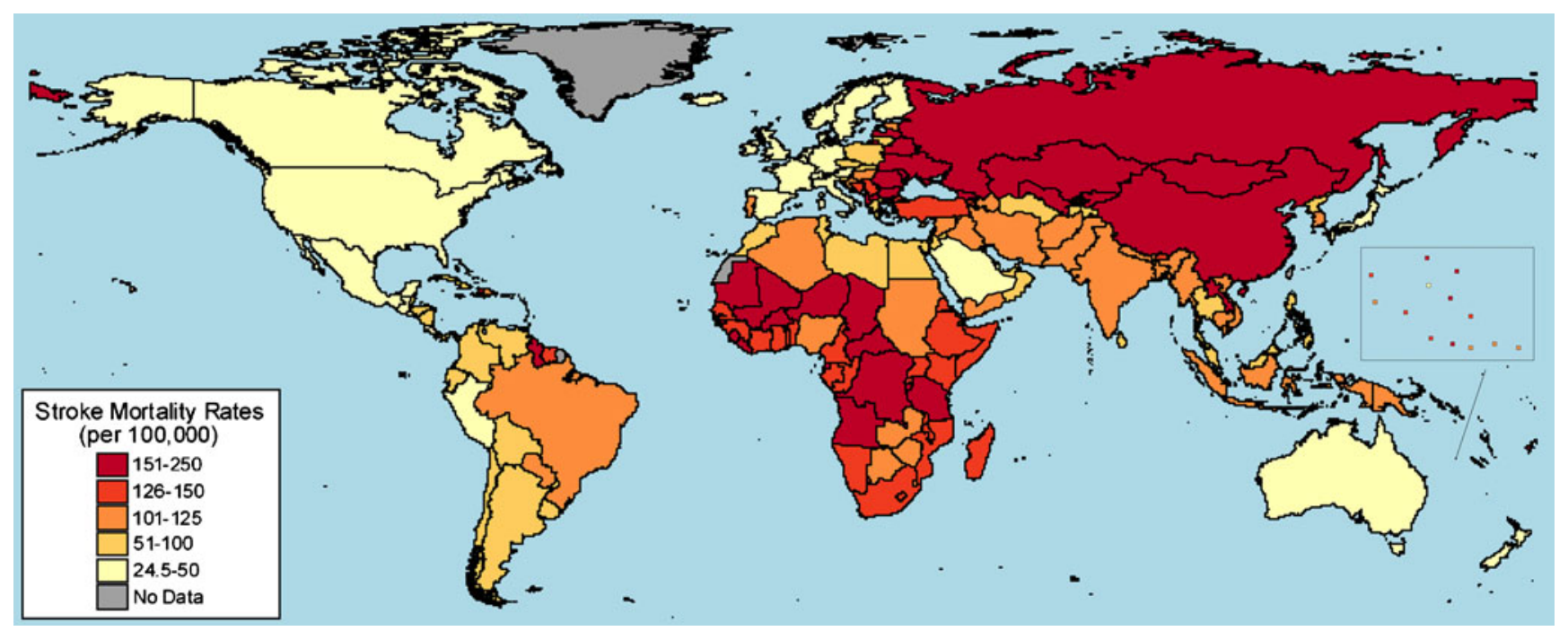

Fig. 1 Age-adjusted and sex-adjusted stroke mortality rates. Rates are highest in Eastern Europe, North Asia, Central Africa, and the South Pacific. (Reproduced with permission from Johnston SC. Global variation in stroke burden and mortality: estimates from monitoring, surveillance, and modeling. Lancet Neurol 2009;8:345-54) 
poorer outcomes in these ethnic groups. Many studies have examined the various risk factors for stroke and their prevalence and control among different racial ethnic groups. Blacks consistently have higher rates of hypertension [70-72]. The diabetes rate was alarmingly high among Native Americans (62\%) [73]. Among others, uncontrolled risk factors can contribute to the poorer outcomes after a stroke.

Administration of thrombolytic therapy, which is associated with better functional outcomes, is highly dependent on the timely arrival at a medical center. There is little data on racial ethnic variations in acute stroke care and outcomes, and the results from available studies are contradictory and inconclusive. Some have reported that being Asian was independently predictive of delay in presentation [74]. Similar studies looking at delays in New York and in the Stroke Belt did not find an association between ethnicity and delay [75, 76], whereas another study conducted in San Diego detected a trend toward delayed presentation among blacks [77]. Also, blacks were less likely to receive intravenous tPA [78, 79]. Although delays in presentation and contraindications were seen more frequently in blacks, these were not statistically significant from whites [78]. Blacks were also less likely to receive care from a neurologist, and studies have found that neurological care has beneficial effects on stroke outcomes [79-81].

Studies have also found ethnic differences in stroke care and work-up during hospitalization that potentially influences short- and long-term outcomes. A large study using Get with the Guidelines database for 1181 hospitals examined the quality of ischemic stroke care received by blacks, whites, and Hispanic stroke patients for a 5-year time span [82]. The study found that blacks received fewer evidencebased care processes than Hispanic or white patients, including lower odds of receiving intravenous thrombolysis, deep vein thrombosis prophylaxis, smoking cessation, discharge anti-thrombotics, anticoagulation for atrial fibrillation, and lipid-lowering therapy. The overall quality of care was observed to improve during the 5-year study period for all ethnic groups.

\section{Trends in Stroke}

In general, the trends in stroke incidence and mortality rates have decreased in the recent decades for high-income countries such as the U.S., the United Kingdom, and Canada [83-86]. Analyses from the Framingham Study revealed a decrease in stroke incidence for the past 50 years, although the lifetime risk of stroke did not decrease to the same degree [86]; this may reflect both an improvement in risk factor control, as well as an improvement in life expectancy. Population-based studies in Oxfordshire (United Kingdom) showed a major reduction in age- and genderspecific stroke incidence for the past 20 years [85]. This decline was found to be associated with an increased use of preventative treatment and better control of vascular risk factors, including hypertension, hyperlipidemia, and smoking. Stroke mortality improvement during 1990 to 2002 was observed in the U.S. and in Canada after mandatory folic acid fortification was in place, but not in England and Wales in which the fortification was not mandatory during the same period [84]. There have also been advancements in acute stroke care and this may have led to a nationwide decrease in the percentage of stroke hospitalizations resulting in death in the U.S. [87]. This decrease was seen regardless of stroke subtypes (Fig. 2).

While there have been reports of decreased stroke incidence and mortality in high-income countries, the opposite has been observed in low-to-middle income countries [83]. A systematic review of population-based studies has found more than $100 \%$ increase in stroke incidence in these countries. Early stroke case fatality is decreasing in both high-income and low-to-middle income countries. However, the overall early stroke case fatality in low-to-middle income countries is found to be $25 \%$ higher than that seen in high-income countries in the past decade. The reasons for these differences are unclear, but low-tomiddle income countries are unlikely to have the same advancements in acute stroke care and secondary stroke prevention measures as seen in high-income countries in the recent decades.

Although advancement in stroke prevention and management has led to many successes in the fight against this disease for the past 2 decades, many challenges remain as clinicians and investigators continue to try and uncover the underlying causes behind the many disparities in stroke burden and outcome observed in epidemiologic studies to be able to design interventions to improve stroke mortality and disability for all.

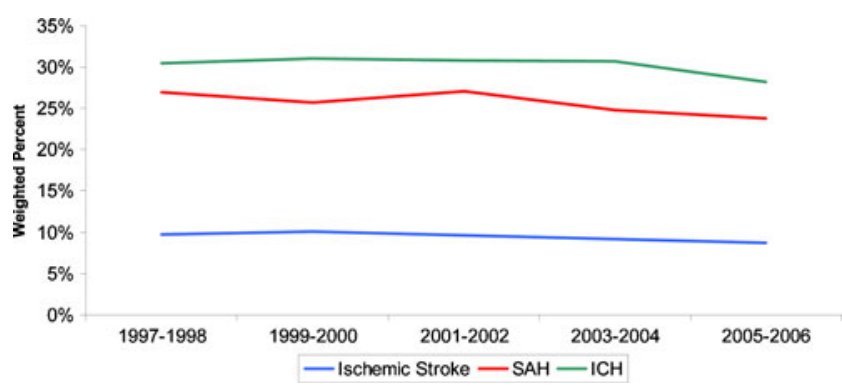

Fig. 2 Distribution of in-hospital stroke mortality rates in the U.S. by stroke subtypes between 1997 and 2006. These trends are statistically significant $(p<0.001)$. (Reproduced with modifications from Ovbiagele B. Nationwide trends in in-hospital mortality among patients with stroke. Stroke 2010;41:1748-1754, with permission from the author) 
Acknowledgements Full conflict of interest disclosure is available in the electronic supplementary material for this article.

\section{References}

1. Roger VL, Go AS, Lloyd-Jones DM, et al. Heart disease and stroke statistics - 2011 update: a report from the american heart association. Circulation 2011;123:e18-e209.

2. Chen C, Hsu C. Stroke: an overview. In: Lisak R, Truong, DD, Caroll, WM, Bhidayasiri R (eds) International neurology: a clinical approach. West Sussex, United Kingdom: WileyBlackwell, 2009:1.

3. Ariesen MJ, Claus SP, Rinkel GJ, Algra A. Risk factors for intracerebral hemorrhage in the general population: a systematic review. Stroke 2003;34:2060-2065.

4. Chong J, Sacco R. Risk factors for stroke, assessing risk, and the mass and high-risk approaches for stroke prevention. In: Gorelick PB, ed. Continuum: Stroke Prevention. Hagerstwon, Maryland: Lippincott Williams and Wilkins, 2005:18-34.

5. Ohene-Frempong K, Weiner SJ, Sleeper LA, et al. Cerebrovascular accidents in sickle cell disease: Rates and risk factors. Blood 1998:91:288-294

6. Ovbiagele B. National sex-specific trends in hospital-based stroke rates. J Stroke Cerebrovasc Dis 2010. doi:10.1016/j.jstroke cerebrovasdis.2010.03.007.

7. Johnston SC, Fayad P, Gorelick PB, et al. Prevalence and knowledge of transient ischemic attacks among us adults. Neurology 2003;60:1429-1434.

8. Brown RD Jr, Petty GW, O'Fallon WM, Wiebers DO, Whisnant JP. Incidence of transient ischemic attack in rochester, minnesota, 1985-1989. Stroke 1998;29:2109-2113.

9. Feigin VL, Shishkin SV, Tzirkin GM, et al. A population-based study of transient ischemic attack incidence in Novosibirsk, Russia, 1987-1988 and 1996-1997. Stroke 2000;31:9-13.

10. Zupping R, Roose M. Epidemiology of cerebrovascular disease in Tartu, Estonia, USSR, 1970 through 1973. Stroke 1976; 7:187-190

11. Dennis MS, Bamford JM, Sandercock PA, Warlow CP. Incidence of transient ischemic attacks in Oxfordshire, England. Stroke 1989;20:333-339.

12. Giroud M, Milan C, Beuriat $P$, et al. Incidence and survival rates during a two-year period of intracerebral and subarachnoid haemorrhages, cortical infarcts, lacunes and transient ischaemic attacks. The stroke registry of dijon: 1985-1989. Int J Epidemiol 1991;20:892-899.

13. Mettinger KL, Soderstrom CE, Allander E. Epidemiology of acute cerebrovascular disease before the age of 55 in the stockholm county 1973-77: I. Incidence and mortality rates. Stroke 1984;15:795-801.

14. Ueda K, Kiyohara Y, Hasuo Y, et al. Transient cerebral ischemic attacks in a Japanese community, Hisayama, Japan. Stroke 1987; 18:844-848

15. Easton JD, Saver JL, Albers GW, et al. Definition and evaluation of transient ischemic attack: A scientific statement for healthcare professionals from the American Heart Association/American Stroke Association Stroke Council; Council on Cardiovascular Surgery and Anesthesia; Council on Cardiovascular Radiology and Intervention; Council on Cardiovascular Nursing; and the Interdisciplinary Council on Peripheral Vascular Disease. The American Academy of Neurology affirms the value of this statement as an educational tool for neurologists. Stroke 2009;40:2276-2293.

16. Ovbiagele B, Kidwcell CS, Saver JL. Epidemiological impact in the United States of a tissue-based definition of transient ischemic attack. Stroke 2003;34:919-924.
17. Giles MF, Rothwell PM. Risk of stroke early after transient ischaemic attack: a systematic review and meta-analysis. Lancet Neurol 2007;6:1063-1072.

18. Johnston SC, Rothwell PM, Nguyen-Huynh MN, et al. Validation and refinement of scores to predict very early stroke risk after transient ischaemic attack. Lancet 2007;369:283-292.

19. Wolf P, D'Agostino RB, Belanger AJ, Kannel WB. Probability of stroke: a risk profile from the Framingham study. Stroke 1991;22:312-318.

20. O'Donnell MJ, Xavier D, Liu L, et al. Risk factors for ischaemic and intracerebral haemorrhagic stroke in 22 countries (The Interstroke Study): a case-control study. Lancet 2010;376:112-123.

21. Elkind MS. Inflammatory mechanisms of stroke. Stroke 2010;41: S3-S8.

22. Lee M, Ovbiagele B. Reno-cerebrovascular disease: linking the nephron and neuron. Expert Rev Neurother 2011;11:241-249.

23. Lewington S, Clarke R, Qizilbash N, Peto R, Collins R. Agespecific relevance of usual blood pressure to vascular mortality: a meta-analysis of individual data for one million adults in 61 prospective studies. Lancet 2002;360:1903-1913.

24. Chobanian A, Bakris GL, Black HR, et al. The seventh report of the joint national committee on prevention, detection, evaluation, and treatment of high blood pressure: The JNC 7 report. JAMA 2003;289:2560-2572.

25. Rothwell PM, Howard SC, Dolan E, et al. Prognostic significance of visit-to-visit variability, maximum systolic blood pressure, and episodic hypertension. Lancet 2010;375:895-905.

26. Wolf PA, Abbott RD, Kannel WB. Atrial fibrillation as an independent risk factor for stroke: The Framingham Study. Stroke 1991;22:983-988.

27. Tayal AH, Tian M, Kelly KM, et al. Atrial fibrillation detected by mobile cardiac outpatient telemetry in cryptogenic TIA or stroke. Neurology 2008;71:1696-1701.

28. Loh E, Sutton MS, Wun CC, et al. Ventricular dysfunction and the risk of stroke after myocardial infarction. $N$ Engl J Med 1997;336:251-257.

29. Folsom AR, Rasmussen ML, Chambless LE, et al. Prospective associations of fasting insulin, body fat distribution, and diabetes with risk of ischemic stroke. The atherosclerosis risk in communities (aric) study investigators. Diabetes Care 1999;22:10771083.

30. Rundek T, Gardener $\mathrm{H}, \mathrm{Xu} \mathrm{Q}$, et al. Insulin resistance and risk of ischemic stroke among nondiabetic individuals from the northern manhattan study. Arch Neurol 2010;67:1195-1200.

31. Boden-Albala B, Sacco RL, Lee HS, et al. Metabolic syndrome and ischemic stroke risk: Northern manhattan study. Stroke 2008;39:30-35.

32. Ovbiagele B, Saver JL, Lynn MJ, Chimowitz M. Impact of metabolic syndrome on prognosis of symptomatic intracranial atherostenosis. Neurology 2006;66:1344-1349.

33. Goldstein LB, Bushnell CD, Adams RJ, et al. Guidelines for the primary prevention of stroke: a guideline for healthcare professionals from the American Heart Association/American Stroke Association. Stroke 2011;42:517-584.

34. Tirschwell DL, Smith NL, Heckbert SR, Lemaitre RN, Longstreth WT Jr, Psaty BM. Association of cholesterol with stroke risk varies in stroke subtypes and patient subgroups. Neurology 2004;63:1868-1875.

35. Norris JW, Zhu CZ, Bornstein NM, Chambers BR. Vascular risks of asymptomatic carotid stenosis. Stroke 1991;22:1485-1490.

36. Abbott AL. Medical (nonsurgical) intervention alone is now best for prevention of stroke associated with asymptomatic severe carotid stenosis: results of a systematic review and analysis. Stroke 2009; 40:e573-e583.

37. Marquardt L, Geraghty OC, Mehta Z, Rothwell PM. Low risk of ipsilateral stroke in patients with asymptomatic carotid stenosis on 
best medical treatment: a prospective, population-based study. Stroke 2010;41:e11-e17.

38. MacClellan LR, Giles W, Cole J, et al. Probable migraine with visual aura and risk of ischemic stroke: The stroke prevention in young women study. Stroke 2007;38:24382445.

39. Lloyd-Jones D, Adams RJ, Brown TM, et al. Heart disease and stroke statistics-2010 update: a report from the American Heart Association. Circulation 2010;121:e46-e215.

40. National Center for Health Statistics. Vital Statistics of the United States, data warehouse. http://www.cdc.gov/nchs/data/dvs/ MortFinal2003_WorkTable307.pdf.

41. Sacco RL, Boden-Albala B, Gan R, et al. Stroke incidence among white, black, and hispanic residents of an urban community: The Northern Manhattan Stroke Study. Am J Epidemiol 1998;147:259268.

42. Lackland DT, Bachman DL, Carter TD, Barker DL, Timms S, Kohli $\mathrm{H}$. The geographic variation in stroke incidence in two areas of the southeastern stroke belt: The Anderson and Pee Dee Stroke Study. Stroke 1998;29:2061-2068.

43. El-Saed A, Kuller LH, Newman AB, et al. Geographic variations in stroke incidence and mortality among older populations in four us communities. Stroke 2006;37:1975-1979.

44. Hollander M, Koudstaal PJ, Bots ML, Grobbee DE, Hofman A, Breteler MM. Incidence, risk, and case fatality of first ever stroke in the elderly population. The Rotterdam Study. J Neurol Neurosurg Psychiatry 2003;74:317-321.

45. Rosamond WD, Folsom AR, Chambless LE, et al. Stroke incidence and survival among middle-aged adults: 9-year follow-up of the atherosclerosis risk in communities (aric) cohort. Stroke 1999;30:736-743.

46. Rosamond W, Flegal K, Friday G, et al. Heart disease and stroke statistics-2007 update: A report from the american heart association statistics committee and stroke statistics subcommittee. Circulation 2007;115:e69-171.

47. Nakayama H, Jorgensen HS, Raaschou HO, Olsen TS. The influence of age on stroke outcome. The Copenhagen Stroke Study. Stroke 1994;25:808-813.

48. Di Carlo A, Lamassa M, Pracucci G, et al. Stroke in the very old: clinical presentation and determinants of 3-month functional outcome: A European perspective. European Biomed Study of Stroke Care Group. Stroke 1999;30:2313-2319.

49. Appelros P, Stegmayr B, Terent A. Sex differences in stroke epidemiology: a systematic review. Stroke 2009;40:10821090.

50. Reeves M, Bhatt A, Jajou P, Brown M, Lisabeth L. Sex differences in the use of intravenous RT-PA thrombolysis treatment for acute ischemic stroke: a meta-analysis. Stroke 2009;40:17431749.

51. Di Carlo A, Lamassa M, Baldereschi M, et al. Sex differences in the clinical presentation, resource use, and 3-month outcome of acute stroke in Europe: aata from a multicenter multinational hospital-based registry. Stroke 2003;34:1114-1119.

52. Kapral MK, Fang J, Hill MD, et al. Sex differences in stroke care and outcomes: Results from the registry of the Canadian Stroke Network. Stroke 2005;36:809-814.

53. Glader EL, Stegmayr B, Norrving B, et al. Sex differences in management and outcome after stroke: a Swedish national perspective. Stroke 2003;34:1970-1975.

54. Holroyd-Leduc JM, Kapral MK, Austin PC, Tu JV. Sex differences and similarities in the management and outcome of stroke patients. Stroke 2000;31:1833-1837.

55. Silva GS, Lima FO, Camargo EC, et al. Gender differences in outcomes after ischemic stroke: role of ischemic lesion volume and intracranial large-artery occlusion. Cerebrovasc Dis 2010;30:470 475 .
56. Gargano JW, Reeves MJ. Sex differences in stroke recovery and stroke-specific quality of life: Results from a statewide stroke registry. Stroke 2007;38:2541-2548.

57. Allen NB, Holford TR, Bracken MB, et al. Trends in one-year recurrent ischemic stroke among the elderly in the USA: 19942002. Cerebrovasc Dis 2010;30:525-532.

58. Johnston SC, Mendis S, Mathers CD. Global variation in stroke burden and mortality: estimates from monitoring, surveillance, and modelling. Lancet Neurol 2009;8:345-354.

59. Howard G, Cushman M, Prineas RJ, et al. Advancing the hypothesis that geographic variations in risk factors contribute relatively little to observed geographic variations in heart disease and stroke mortality. Prev Med 2009;49:129-132.

60. Steenland $\mathrm{K}, \mathrm{Hu} \mathrm{S}$, Walker J. All-cause and cause-specific mortality by socioeconomic status among employed persons in 27 US states, 1984-1997. Am J Public Health 2004;94:10371042.

61. Fukuda Y, Nakamura K, Takano T. Cause-specific mortality differences across socioeconomic position of municipalities in Japan, 1973-1977 and 1993-1998: Increased importance of injury and suicide in inequality for ages under 75 . Int $\mathrm{J}$ Epidemiol 2005;34:100-109.

62. Kapral MK, Wang H, Mamdani M, Tu JV. Effect of socioeconomic status on treatment and mortality after stroke. Stroke 2002;33:268-273.

63. Putman K, De Wit L, Schoonacker M, et al. Effect of socioeconomic status on functional and motor recovery after stroke: A European Multicentre Study. J Neurol Neurosurg Psychiatry 2007;78:593-599.

64. Li C, Hedblad B, Rosvall M, Buchwald F, Khan FA, Engstrom G. Stroke incidence, recurrence, and case-fatality in relation to socioeconomic position: a population-based study of middleaged Swedish men and women. Stroke 2008;39:2191-2196.

65. Lofmark U, Hammarstrom A. Education-related differences in case fatality among elderly with stroke. Neuroepidemiology 2008;31:21-27.

66. Ayala C, Greenlund KJ, Croft JB, et al. Racial/ethnic disparities in mortality by stroke subtype in the United States, 1995-1998. Am J Epidemiol 2001;154:1057-1063.

67. Lisabeth LD, Smith MA, Brown DL, Moye LA, Risser JM, Morgenstern LB. Ethnic differences in stroke recurrence. Ann Neurol 2006;60:469-475.

68. Uchino K, Risser JM, Smith MA, Moye LA, Morgenstern LB. Ischemic stroke subtypes among Mexican Americans and nonHispanic whites: the BASIC project. Neurology 2004;63:574-576.

69. Qureshi AI, Feldmann E, Gomez CR, et al. Consensus conference on intracranial atherosclerotic disease: Rationale, methodology, and results. J Neuroimaging 2009;19(suppl 1):1S-10S.

70. Sacco RL, Hauser WA, Mohr JP, Foulkes MA. One-year outcome after cerebral infarction in whites, blacks, and hispanics. Stroke 1991;22:305-311.

71. Sacco RL, Boden-Albala B, Abel G, et al. Race-ethnic disparities in the impact of stroke risk factors: The Northern Manhattan Stroke Study. Stroke 2001;32:1725-1731.

72. Howard G, Prineas R, Moy C, et al. Racial and geographic differences in awareness, treatment, and control of hypertension: the reasons for geographic and racial differences in stroke study. Stroke 2006;37:1171-1178.

73. Frey JL, Jahnke HK, Bulfinch EW. Differences in stroke between white, Hispanic, and Native American patients: The Barrow Neurological Institute Stroke Database. Stroke 1998;29:29-33.

74. Smith MA, Doliszny KM, Shahar E, McGovern PG, Arnett DK, Luepker RV. Delayed hospital arrival for acute stroke: The Minnesota Stroke Survey. Ann Intern Med 1998;129:190 196. 
75. Goldstein LB, Edwards MG, Wood DP. Delay between stroke onset and emergency department evaluation. Neuroepidemiology 2001;20:196-200.

76. Morris DL, Rosamond W, Madden K, Schultz C, Hamilton S. Prehospital and emergency department delays after acute stroke: The Genentech Stroke Presentation Survey. Stroke 2000;31:25852590.

77. Zweifler RM, Lyden PD, Taft B, Kelly N, Rothrock JF. Impact of race and ethnicity on ischemic stroke. The University Of California At San Diego Stroke Data Bank. Stroke 1995;26:245-248.

78. Johnston SC, Fung LH, Gillum LA, et al. Utilization of intravenous tissue-type plasminogen activator for ischemic stroke at academic medical centers: the influence of ethnicity. Stroke 2001;32:1061-1068.

79. Reed SD, Cramer SC, Blough DK, Meyer K, Jarvik JG. Treatment with tissue plasminogen activator and inpatient mortality rates for patients with ischemic stroke treated in community hospitals. Stroke 2001;32:1832-1840.

80. Goldstein LB, Matchar DB, Hoff-Lindquist J, Samsa GP, Horner RD. VA stroke study: neurologist care is associated with increased testing but improved outcomes. Neurology 2003;61:792-796.
81. Cheng EM, Birbeck G, Vickrey B. VA stroke study: Neurologist care is associated with increased testing but improved outcomes. Neurology 2004;62:1914-1915.

82. Schwamm LH, Reeves MJ, Pan W, et al. Race/ethnicity, quality of care, and outcomes in ischemic stroke. Circulation 2010;121:1492-1501.

83. Feigin VL, Lawes CM, Bennett DA, Barker-Collo SL, Parag V. Worldwide stroke incidence and early case fatality reported in 56 population-based studies: a systematic review. Lancet Neurol 2009;8:355-369.

84. Yang Q, Botto LD, Erickson JD, et al. Improvement in stroke mortality in Canada and the United States, 1990 to 2002. Circulation 2006;113:1335-1343.

85. Rothwell PM, Coull AJ, Giles MF, et al. Change in stroke incidence, mortality, case-fatality, severity, and risk factors in Oxfordshire, UK from 1981 to 2004 (oxford vascular study). Lancet 2004;363:1925-1933.

86. Carandang R, Seshadri S, Beiser A, et al. Trends in incidence, lifetime risk, severity, and 30-day mortality of stroke over the past 50 years. JAMA 2006;296:2939-2946.

87. Ovbiagele B. Nationwide trends in in-hospital mortality among patients with stroke. Stroke 2010;41:1748-1754. 